\title{
Astenia en cáncer avanzado y uso de psicoestimulantes
}

\section{Asthenia in advanced cancer and the use of psychostimulants}

\author{
M.A. Portela ${ }^{1}$, A. Sanz ${ }^{2}$, M. Martínez ${ }^{1}$, C. Centeno ${ }^{1}$
}

\section{RESUMEN}

La astenia es el síntoma más frecuente en enfermos con cáncer avanzado y probablemente, el que más influye en la calidad de vida del paciente oncológico ya que interfiere en su actividad física y social. En la mayor parte de los casos el tratamiento es sintomático. Hay un interés creciente en el uso de psicoestimulantes para el tratamiento de astenia. Metilfenidato y modafinilo son dos psicoestimulantes que han sido ya probados en estudios controlados en astenia del paciente con cáncer avanzado, encontrándose eficaces, particularmente en pacientes con estadíos más avanzados, muy cansados.

Palabras clave. Fatiga. Astenia. Cáncer. Metilfenidato. Modafinilo.

\begin{abstract}
Asthenia is the most frequent symptom in patients with advanced cancer and is probably what most affects the quality of life of oncology patients since it interferes in their physical and social activity. Treatment in the majority of cases is symptomatic. There is growing interest in the use of psychostimulants for treating asthenia. Methylphenidate and modafinil are two psychostimulants that have already been tested in controlled studies on asthenia of the patient with advanced cancer; they have proved to be efficient, particularly in patients in very advanced stages who are very tired.
\end{abstract}

Key words. Fatigue. Asthenia. Cancer. Methylphenidate. Modafinil.
1. Unidad de Medicina Paliativa y Control de Síntomas. Clínica Universidad de Navarra. Pamplona.

2. Sección de Oncología. Hospital Universitario Río Hortega. Valladolid.

Recepción: 1 de abril de 2011

Aceptación provisional: 19 de mayo de 2011

Aceptación definitiva: 15 de junio de 2011

\section{Correspondencia:}

María Angustias Portela

Unidad de Medicina Paliativa y Control de

Síntomas

Clínica Universidad de Navarra

Avda. Pío XII, 36

31008 Pamplona

E-mail: mportela@unav.es 


\section{ASTENIA EN LA POBLACIÓN GENERAL}

La astenia (del griego a: 'no', y sthénos: 'vigor') es un síntoma muy inespecífico, ya que puede aparecer en un sinnúmero de procesos, de base física y psicológica, e incluso presentarse también en la persona sana. De hecho, la astenia es uno de los síntomas más frecuentes también en personas sin patología severa de base, hasta el punto que llega a convertirse en el principal problema clínico para uno de cada diez pacientes de Atención Primaria. La prevalencia de la astenia en el conjunto de la población depende del grado de severidad y de la duración que se estime como cansancio anormal ${ }^{1}$. Así, los estudios muestran que entre el 5 y el $20 \%$ de la población general padece cansancio persistente que interfiere con su actividad diaria.

La vivencia de cansancio depende en parte del significado que le pueda dar la persona que lo padece. De hecho, la astenia y otros síntomas poco específicos producen cierto desencuentro entre el paciente y la actitud del médico. El paciente vivencia su astenia y la interpreta desde su miedo y sus expectativas. El médico, sin embargo, suele mantener una actitud conservadora y expectante ya que es difícil dar una interpretación certera de la astenia y porque son escasas las opciones terapéuticas realmente eficaces, más allá del tratamiento etiológico.

\section{ASTENIA EN EL CÁNCER AVANZADO}

La astenia es el síntoma más frecuente en enfermos con cáncer avanzado y, probablemente, el que más influye en la calidad de vida del paciente oncológico, ya que interfiere a nivel físico, mental, social y económico ${ }^{2-4}$. Sin embargo, hasta ahora no se le ha dado la importancia que merece. Un motivo puede ser que se trata de un síntoma "negativo", carencial, es decir, algo que se pierde con la enfermedad, como la fuerza, la capacidad física o el ánimo, y no algo "positivo", algo nuevo que no se tenía, como el dolor o la disnea. Además, la astenia es un problema que crece en la medida en que el cáncer progresa y en que los tratamientos que no se encuentran dirigidos de manera específica a la causa, son poco eficaces. Y al final, se ve como un síntoma tan "natural" y tan refractario a los tratamientos que no se le presta importancia, siendo infradiagnosticada y como consecuencia, infratratada.

Puede definirse como una sensación subjetiva de cansancio inusual y persistente relacionada con el cáncer o su tratamiento y que interfiere con la capacidad funcional habitual. Los términos españoles cansancio, debilidad o agotamiento se adaptan mejor al concepto inglés "fatigue" que la traducción directa, "fatiga", que es un término ambiguo con acepciones como cansancio, disnea e incluso sensación nauseosa ${ }^{5}$. En general, el síntoma "fatigue" es reconocido por todos los autores como un concepto multidimensional con, al menos, una faceta física y otra cognitiva. El ánimo y también aspectos existenciales y sociales influyen en el modo en que se percibe.

Se trata de un síntoma de origen complejo y multifactorial con una fisiopatología parcialmente conocida. Entre las causas de astenia en el paciente con cáncer avanzado se incluyen las debidas al propio tumor a través de la liberación de citoquinas (proteínas mediadoras de la comunicación intercelular) como interleukina-1, interleukina-6 $\mathrm{y}$ factor de necrosis tumoral alfa o al desarrollo de síndromes paraneoplásicos (incluida la caquexia tumoral), a los tratamientos oncológicos (quimioterapia, radioterapia, inmunoterapia o cirugía) y sus efectos secundarios, a complicaciones del cáncer (anemia, infección, insuficiencia hepática o renal, hipoxia, desregulación vegetativa, deshidratación, alteraciones cardiacas y pulmonares), a alteraciones endocrinas (hipotiroidismo e hipogonadismo), al uso de otras medicaciones (opioides, ansiolíticos, hipnóticos, antihistamínicos, antieméticos o antihipertensivos), a trastornos del sueño, a factores psicológicos (ansiedad, depresión y estrés), a la interrelación con otros síntomas (dolor, nausea o disnea), o a patologías preexistentes (insuficiencia cardiaca, alteraciones neurológicas).

La línea de actuación en la práctica habitual en astenia en enfermos con cáncer se fundamenta en el rastreo sobre la pre- 
sencia del síntoma y su intensidad subjetiva, por ejemplo mediante una escala de 0 a 10. Si la autoevaluación de la astenia alcanza, al menos, un valor 4 sobre 10 se considera relevante y se plantean estudios para detectar causas potencialmente tratables. Si la astenia persiste, bien porque no se encuentran causas susceptibles de tratamiento, bien porque estos tratamientos no son eficaces, es cuando se plantea una actitud de alivio sintomático (Fig. 1).

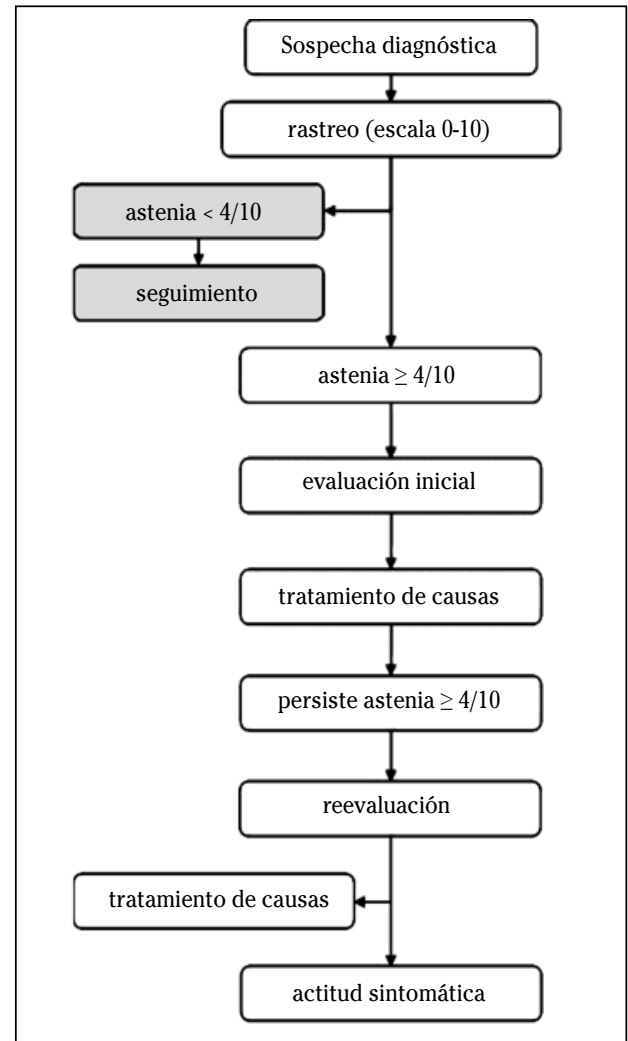

Figura 1. Protocolo de evaluación de astenia en pacientes con cáncer avanzado

En general, en la astenia del paciente oncológico sólo ocasionalmente es posible encontrar una causa que justifique la astenia y para la que exista un tratamiento eficaz: anemia severa, trastornos metabólicos o endocrinos, infecciones... De hecho, en una mayoría de enfermos la única alternativa razonable es la de plantear un tratamiento meramente sintomático.

\section{EVALUACIÓN DE LA ASTENIA}

El síndrome de astenia por cáncer está incluido como entidad en la Clasificación Internacional de Enfermedades CIE-10 (Tabla 1). El definir astenia por cáncer como un síndrome permite documentar la presencia o ausencia de astenia con un lenguaje común, pero presenta la desventaja de disuadir el tratamiento de astenia en aquellos pacientes que no cumplen de forma estricta dichos criterios ${ }^{6}$.

Desde un punto de vista más práctico, el grupo de trabajo de la Asociación Europea de Cuidados Paliativos (EAPC) recomienda que la evaluación sistemática de astenia en un medio no especializado se lleve a cabo con una pregunta única como " ¿se siente usted anormalmente cansado o débil?". Sin embargo, en una Unidad de Oncología o de Cuidados Paliativos la evaluación de astenia y de otros síntomas debería ser sistemática mediante escalas de evaluación general de síntomas. Para fines de investigación o cuando la astenia es un síntoma prioritario, se deberían completar cuestionarios específicos multidimensionales de astenia ${ }^{7}$.

Dentro de los cuestionarios generales, el de Evaluación de Síntomas de Edmonton (ESAS), es un instrumento sencillo y fácil de cumplimentar. Consiste en una hoja impresa que incluye escalas visuales numéricas (0-10) de diez síntomas frecuentes de los enfermos oncológicos, entre ellos astenia ${ }^{8}$. El cuestionario específico de astenia que más se emplea en estudios de oncología recientes es la subescala de fatiga del cuestionario Functional Assessment of Cancer Therapy (FACT-F) que hace una evaluación integrada de los últimos siete días del paciente. El cuestionario general completo FACT utiliza 27 ítems de calidad de vida divididos en cuatro dominios: físico, social, emocional y funcional. Para evaluar astenia la subescala FACT-F incluye 13 ítems específicos. Puede rellenarse como cuestionario autoadministrado o mediante un entrevistador en un tiempo que no debería superar los 5 minutos. El cuestionario FACT y sus diversas subescalas han sido validados en hombres y en mujeres y presentan unos valores aceptables de consistencia interna y de constancia en los resultados al repetir el cuestionario. Existe una versión traducida y validada en castellano ${ }^{9}$. 
Tabla 1. Criterios de la CIE-10 para la fatiga relacionada con el cáncer

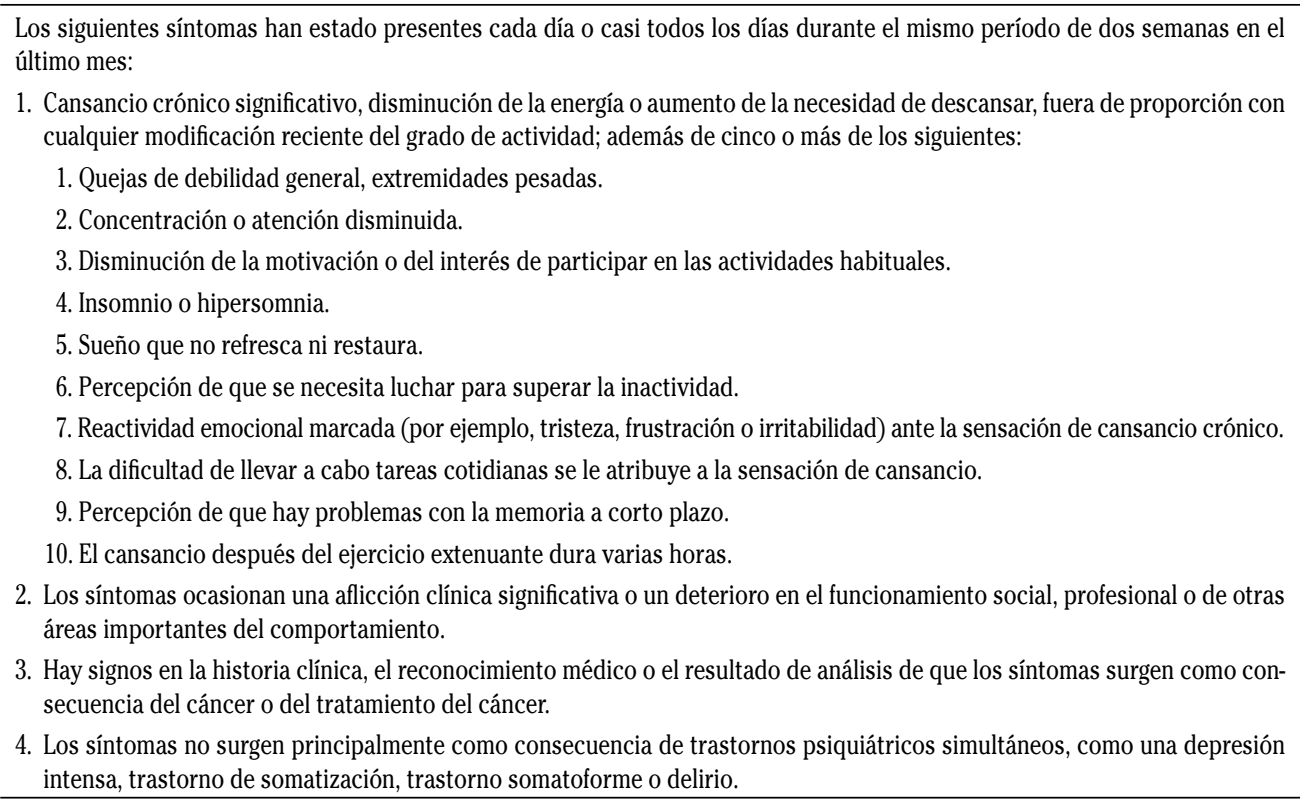

\section{QUÉ SE SABE DEL TRATAMIENTO DE LA ASTENIA EN EL CÁNCER AVANZADO}

La actitud inicial ante la astenia en el enfermo con cáncer avanzado es la de buscar causas que pudieran ser susceptibles de tratamiento etiológico (Fig. 1). Es posible encontrar factores físicos y psicológicos que pueden inducir astenia como anemia, infección, alteraciones de tipo endocrino o metabólico, algunos fármacos, alteraciones del ciclo sueño vigilia o depresión, en los que se puede plantear una línea de actuación específica. Dentro de estas opciones de tratamiento en los últimos años ha cobrado interés el soporte hematológico para paliar el componente de la astenia debido a la anemia ${ }^{10}$.

En la mayor parte de los casos tan sólo es factible plantear un tratamiento sintomático y de soporte que incluye estrategias farmacológicas y no farmacológicas. Entre estas últimas hay algunas que han mostrado cierto beneficio clínico: mejorar la calidad del sueño, apoyo psicológico y terapias complementarias como yoga ${ }^{11}$.
Numerosos estudios han investigado los efectos de la actividad física en la astenia por cáncer. Los resultados de varios metanálisis recientes, encuentran beneficio en el ejercicio, tanto durante como después del tratamiento para el cáncer ${ }^{12-15}$. Se ha sugerido que los cambios producidos por el ejercicio pueden contrarrestar los efectos del tumor y la toxicidad del tratamiento sobre la capacidad física ${ }^{16}$. Hay una gran variedad de programas de ejercicios, no existiendo consenso sobre el tipo e intensidad de ejercicio a realizar.

Entre los fármacos que se han empleado en el tratamiento de la astenia del enfermo oncológico se encuentran: corticoides, progestágenos, inhibidores selectivos de la acetil-colinesterasa (como donezepilo), simpaticomiméticos (como por ejemplo midrodina), testosterona, talidomida y psicoestimulantes (metilfenidato).

Los corticoides como la prednisona o la dexametasona se han usado con frecuencia, si bien la evidencia que avala su eficacia es escasa ${ }^{17}$.

Entre los tratamientos de tipo hormonal, los progestágenos muestran una acti- 
vidad muy limitada. Bruera y col en un ensayo doble-ciego comparado con placebo, mostraron que $460 \mathrm{mg}$ diarios de acetato de megestrol tienen un efecto beneficioso en los niveles de actividad después de 10 días de tratamiento ${ }^{18}$.

El donezepilo es un inhibidor selectivo de la acetilcolinesterasa, que se emplea en la enfermedad de Alzheimer. Este fármaco actuaría incrementando la actividad colinérgica central, que paliaría la insuficiencia vegetativa por disminución de la producción colinérgica y adrenérgica periférica de los pacientes con astenia y cáncer avanzado. Sin embargo, los resultados del estudio de Bruera y col no demostraron ventaja frente a placebo ${ }^{19}$.

El hipogonadismo (niveles de testosterona bajos) parece ser frecuente en pacientes con cáncer avanzado y se asocia con alteraciones del ánimo, astenia y anorexiacaquexia. La testosterona en forma de parches transdérmicos o gel pudiera ser una opción ${ }^{20}$.

La talidomida es inhibidor del factor de necrosis tumoral (TNF alfa) y modula las interleuquinas. Bruera y col en su estudio sobre caquexia afirman que la talidomida puede tener también beneficio para astenia en cáncer ${ }^{21}$.

Recientemente se ha publicado un estudio randomizado, controlado con placebo, sobre el tratamiento de astenia por cáncer con ginseng americano, una sustancia obtenida de la raíz de la planta herbácea Panax quinquefolium con propiedades estimulantes físicas y mentales. No se encontraron diferencias significativas, si bien se observó cierta actividad con adecuada tolerancia a dosis de 1000-2000 mg diarios ${ }^{22}$.

\section{USO DE PSICOESTIMULANTES EN EL TRATAMIENTO DE LA ASTENIA EN EL CÁNCER AVANZADO}

Los psicoestimulantes son fármacos capaces de estimular la conducta, por un mecanismo que implica la reducción del umbral de los sistemas de alerta o vigilia, de modo que el individuo se encuentre en situación de responder con más facilidad o prontitud a los estímulos exógenos y endógenos.

El uso de algunos estimulantes, como la cafeína, está muy extendido en la población general. Actúa bloqueando los receptores de adenosina promotores del sueño. Pero existen además otros fármacos de este tipo comercializados, como metilfenidato y modafinilo que actúan incrementando los niveles de dopamina en el sistema nervioso central. La dopamina tiene un rol importante en los sistemas mesolímbico y mesocortical, los cuales están en relación con la motivación y la atención. Estos efectos son mediados por los receptores dopaminérgicos. El metilfenidato bloquea estos receptores en distintas áreas cerebrales y el modafinilo actúa inhibiendo los transportadores de dopamina en áreas cerebrales específicas que regulan el ritmo sueñovigilia $^{23-24}$.

Las indicaciones aprobadas para metilfenidato son déficit de atención e hiperactividad en niños y adolescentes y narcolepsia, y las del modafinilo, somnolencia diurna en pacientes con narcolepsia, trastornos del sueño por turnos de trabajo y apnea obstructiva del sueño con somnolencia excesiva residual a pesar del uso óptimo de presión positiva continua. Su uso fuera de indicación está extendido en oncología, para tratar distintos síntomas como desánimo cuando el pronóstico de vida es menor de 3 meses, somnolencia por opioides, delirium hipoactivo, secuelas en niños tratados con quimioterapia por cánceres infantiles (principalmente tumores cerebrales y leucemia linfoide aguda) y astenia.

El uso de psicoestimulantes fuera de las indicaciones autorizadas se rige por el Real Decreto 1015/200925. Según esta normativa el facultativo que asista al paciente debe obtener del mismo el correspondiente consentimiento informado, justificar en la historia clínica la necesidad del uso del medicamento, respetar las prescripciones establecidas en relación con la prescripción y/o dispensación y el protocolo terapeútico asistencial del centro sanitario y notificar las sospechas de reacciones adversas $^{26}$. 
Tabla 1. Estudios comparativos con distribución aleatoria con metilfenidato en astenia por cáncer

\begin{tabular}{|c|c|c|c|c|c|c|c|}
\hline Autor y año & $\begin{array}{c}\text { Tipo de } \\
\text { enfermedad }\end{array}$ & Nivel de astenia & $\begin{array}{c}\text { Dosis diaria } \\
\text { y pauta de } \\
\text { administración }\end{array}$ & $\begin{array}{c}\text { Duración } \\
\text { del } \\
\text { tratamiento }\end{array}$ & $\begin{array}{c}\mathbf{n} \\
\text { randomizado } \\
(\mathrm{n} \text { final) }\end{array}$ & $\begin{array}{c}\text { Resultados (cambios } \\
\text { en variable principal } \\
\text { metilfenidato/ } \\
\text { placebo) }\end{array}$ & Efectos adversos \\
\hline $\begin{array}{l}\text { Bruera E y } \\
\text { col }(2006)^{33}\end{array}$ & Cáncer avanzado & $\mathrm{EVN} \geq 4$ & $\begin{array}{l}5 \mathrm{mg} \text { a demanda } \\
\text { hasta cada } 2 \mathrm{~h} \\
\text { (Máximo } \\
20 \mathrm{mg} / \text { día) }\end{array}$ & 8 días & $112(105)$ & $\begin{array}{l}\text { FACT -F } 9,6 / 7,5 \\
(p=0,31) \\
\text { ESAS -2,7/-1,9 } \\
(p=0,14)\end{array}$ & $\begin{array}{l}\text { Insomnio, } \\
\text { anorexia e } \\
\text { inquietud }\end{array}$ \\
\hline $\begin{array}{l}\text { Butler JM y } \\
\operatorname{col}(2007)^{34}\end{array}$ & $\begin{array}{l}\text { Cáncer cerebral } \\
\text { en tratamiento } \\
\text { con radioterapia }\end{array}$ & $\begin{array}{l}\text { Cualquier nivel } \\
\text { de astenia (La } \\
\text { medicación se } \\
\text { usa de forma } \\
\text { profiláctica). }\end{array}$ & $\begin{array}{l}5 \text { mg dos veces } \\
\text { al día } \\
\text { (Máximo } \\
30 \mathrm{mg} / \text { día) }\end{array}$ & 8 semanas & $68(32)$ & $\begin{array}{l}\text { FACT-F } 33,7 / 35,6 \\
(\mathrm{p}=0,64)\end{array}$ & $\begin{array}{l}\text { Náuseas y } \\
\text { vómitos }\end{array}$ \\
\hline $\begin{array}{l}\text { Mar Fan y } \\
\text { col }(2008)^{35}\end{array}$ & $\begin{array}{l}\text { Cáncer mama en } \\
\text { tratamiento con } \\
\text { quimioterapia }\end{array}$ & $\begin{array}{l}\text { Cualquier nivel } \\
\text { de astenia }\end{array}$ & $\begin{array}{l}5 \mathrm{mg} \text {, dos veces } \\
\text { al día } \\
\text { (Máximo } \\
20 \mathrm{mg} / \text { día) }\end{array}$ & 12 semanas & $57(39)$ & $\begin{array}{l}\text { FACT-F } 10,1 / 11,5 \\
(\mathrm{p}=0,95)\end{array}$ & $\begin{array}{l}\text { Ansiedad, } \\
\text { insomnio y } \\
\text { mareo }\end{array}$ \\
\hline $\begin{array}{l}\text { Lower EE y } \\
\text { col }(2009)^{36}\end{array}$ & $\begin{array}{l}\text { Cáncer mama } \\
\text { y ovario } \\
\text { principalmente } \\
\text { tras tratamiento } \\
\text { con quimioterapia }\end{array}$ & $\begin{array}{l}\text { Astenia según } \\
\text { criterios ICD-10 }\end{array}$ & $\begin{array}{l}5 \mathrm{mg} \text {, dos veces } \\
\text { al día } \\
\text { (Máximo } \\
50 \mathrm{mg} / \text { día) }\end{array}$ & 8 semanas & $154(152)$ & $\begin{array}{l}\text { FACT-F - } 10,5 /-6,8 \\
(\mathrm{p}=0.02)\end{array}$ & $\begin{array}{l}\text { Cefalea, náuseas } \\
\text { y boca seca }\end{array}$ \\
\hline $\begin{array}{l}\text { Roth AJ y } \\
\operatorname{col}(2010)^{37}\end{array}$ & $\begin{array}{l}\text { Cáncer próstata } \\
\text { avanzado }\end{array}$ & $\mathrm{EVN} \geq 4$ & $\begin{array}{l}5 \mathrm{mg} / \text { día } \\
\text { (Máximo } \\
30 \mathrm{mg} / \text { día) }\end{array}$ & 6 semanas & $32(23)$ & $\begin{array}{l}\text { BFI } 3,5 / 1,1 \\
(p=0,03)\end{array}$ & $\begin{array}{l}\text { Taquicardia e } \\
\text { hipertensión }\end{array}$ \\
\hline $\begin{array}{l}\text { Moraska } \\
\text { AR y col } \\
(2010)^{27}\end{array}$ & $\begin{array}{l}\text { Cáncer en } \\
\text { tratamiento con } \\
\text { quimioterapia, } \\
\text { radioterapia o } \\
\text { terapia biológica }\end{array}$ & $\mathrm{EVN} \geq 4$ & $\begin{array}{l}18 \text { mg / día } \\
\text { (Máximo } \\
54 \text { mg /día) }\end{array}$ & 4 semanas & $148(125)$ & $\begin{array}{l}\text { BFI }(\mathrm{p}=0,32) \\
\text { BFI subgrupo } \\
\text { estadios avanzados: } \\
19,7 / 2,1(\mathrm{p}=0,02)\end{array}$ & $\begin{array}{l}\text { Nerviosismo y } \\
\text { anorexia }\end{array}$ \\
\hline
\end{tabular}

*FACT-F Functional Assessment of Cancer Therapy - Fatigue; ESAS, Edmonton Sympton Assessment Scale; BFI, Brief Fatigue Inventory ${ }^{*}$ Criterios ICD-10: International Classification of Disease, Tenth Revision criteria for cancer-related fatigue

Hay interés creciente en el uso de psicoestimulantes para el tratamiento de astenia tanto en pacientes con enfermedad avanzada como en aquellos que reciben tratamientos oncológicos (quimioterapia y radioterapia). Los últimos estudios apuntan a que el perfil de pacientes más beneficiados es el de aquellos con estadios más avanzados de cáncer y astenia intensa ( $\geq 7$ en una escala 0 a 10), particularmente los que responden positivamente el primer día de tratamiento ${ }^{27-29}$.

Los psicoestimulantes están contraindicados cuando hay antecedentes de enfermedades cardiovasculares como hipertensión severa, arritmias y angor, enfermedades psiquiátricas tipo ansiedad, agitación, psicosis $\mathrm{y}$ adicciones, epilepsia, hipertiroidismo y glaucoma de ángulo estrecho. El modafinilo no tiene contraindicación en hipertiroidismo ni glaucoma. También está contraindicado su asociación con simpaticomiméticos como los inhibidores de monoamino oxidasa (IMAO) por el riesgo de crisis hipertensivas. En general, son fármacos bien tolerados. Los efectos secundarios más frecuentes con metilfenidato son inquietud, insomnio y sequedad de boca ${ }^{30}$, si bien la proporción de pacientes que abandona el tratamiento por este tipo de efectos es muy escasa. Respecto al modafinilo se describen efectos secundarios similares, aparte de cefalea y mareo ${ }^{31-32}$. La hiporexia es un efecto secundario muy infrecuente. 
Tabla 2. Estudios comparativos con distribución aleatoria con modafinilo en astenia por cáncer

\begin{tabular}{|c|c|c|c|c|c|c|c|}
\hline Autor y año & $\begin{array}{c}\text { Tipo de } \\
\text { enfermedad }\end{array}$ & Nivel de astenia & Dosis diaria & $\begin{array}{c}\text { Duración del } \\
\text { tratamiento }\end{array}$ & $\begin{array}{c}\mathrm{n} \\
\text { randomizado } \\
(\mathrm{n} \text { final) }\end{array}$ & Resultados & $\begin{array}{c}\text { Efectos } \\
\text { adversos }\end{array}$ \\
\hline $\begin{array}{l}\text { Kaleita TA y } \\
\text { col }(2006)^{31}\end{array}$ & $\begin{array}{l}\text { Tumores } \\
\text { cerebrales en } \\
\text { tratamiento } \\
\text { con cirugía, } \\
\text { quimioterapia } \\
\text { o radioterapia }\end{array}$ & $\begin{array}{l}\text { Astenia y /o } \\
\text { alteración de } \\
\text { la atención } \\
\text { ó memoria } \\
\text { moderada } \\
\text { o severa } \\
\text { (Clinical Global } \\
\text { Impression of } \\
\text { Severity ratings) }\end{array}$ & $200-400 \mathrm{mg}$ & 56 días & 30 (NR) & $\begin{array}{l}\text { - FSS (Modafinilo) } \\
\text { 5,2-3,6 (p<0,0001) } \\
\text { - MFIS (Modafinilo) } \\
\text { 50,2-30,5 ( }<< \\
\text { 0,0001) } \\
\text { - VAFS (Modafinilo) } \\
\text { 4,0-6,7 (p=0,0001) }\end{array}$ & $\begin{array}{l}\text { Cefalea } \\
\text { Insomnio } \\
\text { Mareo } \\
\text { Boca seca }\end{array}$ \\
\hline $\begin{array}{l}\text { Lundorff } \\
\text { LE y col } \\
(2009)^{32}\end{array}$ & $\begin{array}{l}\text { Cáncer } \\
\text { avanzado }\end{array}$ & $\begin{array}{l}\text { Astenia > 5/10 } \\
(\text { ESAS) }\end{array}$ & $200 \mathrm{mg}$ & $\begin{array}{l}4 \text { días cada } \\
\text { periodo } \\
\text { (crossover) }\end{array}$ & 28 & $\begin{array}{l}\text { - ESAS fatigue } \\
\text { (Modafinilo- } \\
\text { Placebo): }-0,857 \\
\text { (p=0.111) }\end{array}$ & $\begin{array}{l}\text { Náuseas } \\
\text { Cefalea } \\
\text { Inquietud }\end{array}$ \\
\hline $\begin{array}{l}\text { Jean-Pierre P } \\
\text { y col }(2010)^{28}\end{array}$ & $\begin{array}{l}\text { Cáncer en } \\
\text { tratamiento } \\
\text { con } \\
\text { quimioterapia }\end{array}$ & $\begin{array}{l}\text { Astenia }^{3} 2 / 10 \\
(\mathrm{BFI})\end{array}$ & $100-200 \mathrm{mg}$ & 44 días & 877 (544) & $\begin{array}{l}\text { - BFI-3 en astenia } \\
\text { severa }(\geq 7)-1,31 \\
(\mathrm{p}=0,033)\end{array}$ & $\begin{array}{l}\text { Cambios de } \\
\text { humor } \\
\text { Arritmia } \\
\text { cardiaca } \\
\text { Cefalea } \\
\text { Mareo }\end{array}$ \\
\hline
\end{tabular}

*FSS indica Fatigue Severity Scale; VAFS, Visual Analogue Fatigue Scale; MFIS, Modified Fatigue Impact Scale; ESAS, Edmonton Symptom Assessesment Scale; BFI, Brief Fatigue Inventory

El metilfenidato es el psicoestimulante más estudiado para tratar astenia por cáncer $^{33-37}$. Sin embargo, el modafinilo podría presentar algunas ventajas como menor excitabilidad, menor fenómeno de tolerancia y mínimo potencial de abuso (al no actuar directamente sobre los receptores dopaminérgicos y hacerlo en áreas específicas). Se necesita más experiencia en su uso. En la tabla 1 se presentan seis estudios aleatorizados con metilfenidato. Todos son estudios recientes e incluyen pacientes con distintas patologías oncológicas y cualquier nivel de astenia o al menos astenia moderada. En general, la dosis de inicio fue de $5 \mathrm{mg}$ dos veces al día ajustándose hasta un máximo de $50 \mathrm{mg}$. Para evaluar los resultados se utilizaron la escala Edmonton Sympton Assessment Scale (ESAS) y los cuestionarios Functional Assessment of Cancer Therapy - Fatigue (FACT) y Brief Fatigue Inventory (BFI). Dos estudios fueron positivos encontrando significativo el alivio de astenia en el grupo de metilfenidato. En el estudio de Mo- raska y $\mathrm{col}^{27}$ el resultado fue positivo sólo en el subgrupo de pacientes con estadíos más avanzados de la enfermedad. En la tabla 2 se presentan los tres estudios aleatorizados disponibles con modafinilo, usando dosis entre 100 y $400 \mathrm{mg}$. El estudio más reciente de Jean Pierre y coll $^{28}$ reclutó 877 enfermos en tratamiento con quimioterapia, encontrando mejoría significativa con modafinilo respecto a placebo en el subgrupo de pacientes con astenia más severa $\left({ }^{3} 7 / 10\right)$. Será interesante, en un futuro, poder disponer de estudios que comparen resultados de metilfenidato frente a modafinilo.

Existen tres presentaciones de metilfenidato, una de liberación inmediata Rubifen ${ }^{\circledR}(5$ y $10 \mathrm{mg})$ y dos de liberación sostenida Concerta ${ }^{\circledR}(18,36$ y $54 \mathrm{mg})$ con una duración de 10 a 12 h, y Medikinet ${ }^{\circledR}$ (20, 30 y $40 \mathrm{mg}$ ), con una duración de $8 \mathrm{~h}$. Del modafinilo existen comercializadas dos, Modiodal ${ }^{\circledR}$ de 100 y $200 \mathrm{mg}$. El tratamiento medio diario es más económico con metilfenidato que con modafinilo. 
Para el alivio de astenia en cáncer, la dosis inicial de metilfenidato es de $5 \mathrm{mg}$ dos veces al día, en la mañana y al mediodía con el fin de evitar interferir en el sueño, teniendo en cuenta que su semivida es de 2-4 h. Dosis superiores a $30 \mathrm{mg}$ diarios no han aportado beneficio. Para el modafinilo, la dosis inicial es de $100 \mathrm{mg}$, una vez al día en la mañana, con una semivida en torno a las $12 \mathrm{~h}$. Al igual que con el metilfenidato, a partir de ahí se puede realizar un ajuste individual. Con el modafinilo es habitual aumentar la dosis hasta los $200 \mathrm{mg}$ diarios para encontrar la máxima eficacia.

En definitiva, metilfenidato y modafinilo son dos psicoestimulantes que han sido ya probados en estudios controlados en astenia del paciente con cáncer avanzado, encontrándose eficaces, particularmente en pacientes con estadíos más avanzados, muy cansados. Estos datos son preliminares y aún insuficientes para sentar indicación, pero el clínico que atiende a pacientes con cáncer avanzado debería estar familiarizado con su uso, pues podrían ser fuente de alivio cuando fallan otros recursos. Son fármacos bien tolerados y aunque tienen un perfil bastante similar, disponer de varios psicoestimulantes permite tener alternativas cuando uno de ellos falla o está contraindicado.

\section{BIBLIOGRAFÍA}

1. Sharpe M, Wilks D. Fatigue. BMJ 2002; 325 : 480-483.

2. Vogelzang NJ, Breitbart W, Cella D, Curt GA, Groopman JE, Horning SJ et al. Patient, caregiver, and oncologist perceptions of cancer-related fatigue: results of a tripart assessment survey. The Fatigue coalition. Semin Hematol 1997; 34: 4-12.

3. Curt GA, Breitbart W, Cella D, Groopman JE, HoRnING SJ, ITRI LM et al. Impact of cancerrelated fatigue on the lives of patients: new findings from the Fatigue Coalition. Oncologist 2000; 5: 353-360.

4. Gónzalez Barón M, Feyjóo M, Carulla TJ, Camps C, Escobar Y, Belda-Iniesta E. Study of the prevalence of tumor-related asthenia in Spanish cancer patients. Clin Transl Oncol 2008; 10: 351-358.

5. Centeno C, Portela MA, Carvajal A, San Miguel MT, Urdiroz J, Ramos L et al. What is the best term in Spanish to express the concept of cancer-related fatigue? J Palliat Med 2009; 12: 441-445.

6. Cella D, Davis K, Breitbart W, Curt G. CancerRelated Fatigue: Prevalence of proposed diagnostic criteria in a United States sample of cancer survivors. J Clin Oncol 2001; 19: 3385-3391.

7. Radbruch L, Stresser F, Elsner F, Gonzalves JF, LogE J, KAASA $S$ et al. Fatigue in palliative care patients - an EAPC approach. Palliat Med 2008; 22: 13-32.

8. Bruera E, Kuehn N, Miller MJ, Selmser P, MacmiLLAN K. The Edmonton Symptom Assessment System (ESAS): a simple method for the assessment of palliative care patients. J Palliat Care 1991; 7: 6-9.

9. Yellen SB, Cella DF, Webster K, Blendowski C, KAPLAN E. Measuring fatigue and others anemia-related symptoms with the Functional Assessment of Cancer Therapy (FACT) measurement system. J Pain Symtom Manage 1997; 13: 63-74.

10. Rizzo JD, Brouwers M, Hurley P, Seidenfeld J, Arcasoy MO, SpIVACK JL et al. American Society of Clinical Oncology/American Society of Hematology clinical practice guideline update on the use of epoetin and darbepoetin in adult patients with cancer. J Clin Oncol 2010; 28: 4996-5010.

11. Wanchai A, Armer JM, Stewart BR. Nonpharmacologic supportive strategies to promote quality of life in patients experiencing cancer-related fatigue: a systematic review. Clin J Oncol Nurs 2011; 15: 203-214.

12. CRAmp F, Daniel J. Exercise for the management of cancer - related fatigue in adults. Cochrane Database Syst Rev 2008; 16: CD006145.

13. Speck RM, Courneya KS, Mâsse LC, Duval S, SchMITZ KH. An update of controlled physical activity trials in cancer survivors: a systematic review and meta-analysis. J Cancer Surviv 2010; 4: 87-100.

14. Velthuis MJ, Agasi-Idenburg SC, Aufdemkampe G, WitTink HM. The effect of physical exercise on cancer-related fatigue during cancer treatment: a meta-analysis of randomised controlled trials. Clin Oncol (R Coll Radiol) 2010; 22: 208-221.

15. Brown JC, Huedo-Medina TB, Pescatello LS, PesCATEllo SM, FerRer RA, JohnSON BT. Efficacy of exercise interventions in modulating cancerrelated fatigue among adult cancer survivors: a meta-analysis. Cancer Epidemiol Biomarkers Prev 2011; 20: 123-133. 
16. Dimeo F. Radiotherapy-related fatigue and exercise for cancer patients: a review of the literature and suggestions for future research. Front Radiat Ther Onco 2002; 37: 4956.

17. Bruera E, Roca E, Cedaro L, Carraro S, Chacon R. Action of oral methylprednisolone in terminal cancer patients: a prospective randomized double-blind study. Cancer Treat Rep 1985; 69: 751-754.

18. Bruera E, Ernst S, Hagen N, Spachynski K, Belzile $\mathrm{M}$, HANSON $\mathrm{J}$ et al. Effectiveness of megestrol acetate in patients with advanced cancer: a randomized, double-blind, crossover study. Cancer Prev Control 1998; 2: 74-78.

19. Bruera E, El Osta B, Valero V, Driver lC, Pei BL, SHEN L et al. Donepezil for cancer fatigue. A double-blind, randomized, placebo-controlled trial. J Clin Oncol 2007; 25: 3475-3481.

20. Strasser F, Palmer JL, Schover LR, Yusuf SW, Pisters K, Vassilopoulou-Sellin R et al. The Impact of hypogonadism and autonomic dysfunction on fatigue, emotional function and sexual desire in male patients with advanced cancer. Cancer 2006; 107: 2949-2957.

21. Bruera E, Neumann CM, Pituskin E, Calder K, BALL G, HANSON J et al. Thalidomide in patients with cachexia due to terminal cancer: preliminary report. Ann Oncol 1999; 10: 857-859.

22. Barton DL, Soori GS, Bauer BA, Sloan JA, JoHNSON PA, Figueras $\mathrm{C}$ et al. Pilot study of Panax quinquefolius (American ginseng) to improve cancer-related fatigue: a randomized, double-blind, dose-finding evaluation: NCCTG trial N03CA. Support Care Cancer 2010; 18: 179-187.

23. Minzenderg MJ, CARTER CS. Modafinil: A review of neurochemical actions and effects on cognition: Neuropsychopharmacol 2008; 33: 1477-1502.

24. Engberg TM, Dennis SA, Jones MS, Miller MS, CoNTRERAS PC. Brain regional substrates for the actions of the novel wake-promoting agent modafinil in the rat: comparison with amphetamine. Neuroscience 1998; 87: 905-911.

25. Real Decreto 1015/2009, de 19 de junio, por el que se regula la disponibilidad de medicamentos en situaciones especiales (BOE, 20 de julio).

26. Barrios Flores LF. Nueva legislación en torno al uso compasivo del medicamento. Oncologyplus 2011; 1: 30-41.

27. Moraska AR, Sood A, Dakhil SR, Sloan JA, Barton D, Atherton PJ et al. Phase III, randomized, double-blind, placebo-controlled study of long-acting methylphenidate for cancer-related fatigue: North Central Cancer
Treatment group NCCTG-N05C7 Trial. J Clin Oncol 2010; 28: 3673-3679.

28. Jean-Pierre P, Morrow GR, Roscoe JA, Heckler $\mathrm{CH}_{\text {, Mohile }} \mathrm{S}$, JANelsins $\mathrm{M}$ et al. A phase 3 randomized, placebo-controlled, double-blind, clinical trial of the effect of modafinil on cance-related fatigue among 631 patients receiving chemotherapy. Cancer 2010; 116: 3513-3520.

29. Yennurajalingam S, Palmer JL, Chacko R, BrueRA E. Factors associated with response to methylphenidate in advanced cancer patients. Oncologist 2011; 16: 246-253.

30. Lasheen W, Walsh D, Mahmoud F, Davis MP, RIVERA N, KHOSHKNABI DS. Methylphenidate side effects in advanced cancer: a retrospective analysis. Am J Hosp Palliat Care 2010; 27: 1623.

31. Kaleita TA, Wellisch DK, Graham CA, Steh B, NGhiemphu P, Ford JM ET al. Pilot study of modafinil for treatment of neurobehavioral dysfunction and fatigue in adult patients with brain tumors. J Clin Oncol 2006; 24: 1503.

32. LundorfF LE, Jonsson BH, SJogren P. Modafinil for attentional and psychomotor dysfunction in advanced cancer: a double-blind, randomised, cross-over trial. Palliat Med 2009; 23: 731-738.

33. Bruera E, Valero V, Driver L, Shen L, Willey J, Zhang $\mathrm{T}$ et al. Patient-controlled methylphenidate for cancer fatigue: A double-blind, randomized, placebo- controlled trial. J Clin Oncol 2006; 24: 2073-2078.

34. Buttler JM, Case LD, Atkins J, Frizzell B, SANDERS G, GRIFFIN P et al. A phase III, double-blind, placebo-controlled prospective randomized clinical trial of d-threo-methylphenidate $\mathrm{HCI}$ in brain tumor patients receiving radiation therapy. Int J Radiat Oncol Biol Phys 2007; 69: 1496-1501.

35. Mar Fan HG, Clemons M, Xu W, Chemerynsky I, Breunis H, Braganza S et al. A randomized, placebo-controlled, double-blind trial of the effects of d-methylphenidate on fatigue and cognitive dysfunction in women undergoing adjuvant chemotherapy for breast cancer. Support Care Cancer 2008; 16: 577-583.

36. Lower EE, Fleishman S, Cooper A, Zeldis J, Faleck $\mathrm{H}, \mathrm{Yu} \mathrm{Z}$ et al. Efficacy of dexmethylphenidate for the treatment of fatigue after cancer chemotherapy: a randomized clinical trial. J Pain Symptom Manage 2009; 38: 650-662.

37. Roth AJ, Nelson C, Rosenfeld B, Scher H, Slovin S, Morris M et al. Methylphenidate for fatigue in ambulatory men with prostate cancer. Cancer 2010; 116: 5102-5110. 